\title{
Aproximación filosófica del concepto de fe en la teología de Karl Rahner: aplicaciones y actualidad
}

\author{
Maynor Barrientos Amador \\ Investigador del Programa de Investigación para la Promoción del Trabajo en Red de la UNED-Costa Rica (ProRed). Máster en \\ Teología Católica de la UNED y Máster de Literatura en Inglés de la Universidad de Costa Rica. Correo electrónico: mbarrientos@, \\ uned.ac.cr
}

\author{
Recibido: 15 de marzo 2013 • Aprobado: 19 de julio 2013
}

\section{RESUMEN}

Este trabajo pretende delinear los principales aportes del teólogo alemán Karl Rahner al concepto de la fe con base en una revisión de su herencia teológica y filosófica. El concepto de fe en Rahner está marcado por la influencia del pensamiento místico jesuita, el pensamiento filosófico del idealismo alemán y el trabajo revisionista de los teólogos afines a la "nueva teología".

Palabras Claves: Fe; Karl Rahner; Teología; Cristianismo; Iglesia Católica.

\section{ABSTRACT}

This essay highlights the main contributions done to the concept of faith by the German Theologian Karl Rahner based on a revision of his theological and philosophical heritage. Rahner's concept of faith is highly influenced by the Jesuits' mystic thought, German idealism and the revisionist work of theologian known as "the new theology".

Keywords: Faith; Karl Rahner; Theology; Christianity; Catholic Church.

\section{Introducción}

Después de Pablo de Tarso, la discusión sobre el concepto de fe se limitó básicamente al alcance del debate entre fe y razón y, dentro de esta disputa, el concepto paulino de fe fue, generalmente, usado como ejemplo de qué tan diferentes son las vías de acceso al conocimiento de Dios y su acción en el mundo, ya sea por medio de la acción divina (fe), o a través de la razón humana (Concilio Vaticano I, en The Christian Theology Reader, p. 38). Así pues, en realidad, aunque la razón humana puede dar cuenta de la existencia y la acción de Dios en el mundo, Él permanece en la oscuridad de la razón y es por medio de la fe que este espacio de oscuridad puede abrirse a la humanidad. Por consiguiente, la discordia entre fe y razón a partir de Pablo y la reflexión grecoromana posterior, hasta nuestros días, se centra en tres áreas, cada una de las cuales opone la fe y la razón como fuentes de conocimiento de Dios y su acción en el mundo:

1. La oposición entre fe y razón se da en relación con la existencia de Dios y de qué se denomina como existencia de Dios, planteándose la pregunta sobre qué tipo de realidad es Dios; 
esta discusión se da, entonces, en el área de desarrollo de la ontología;

2. La oposición entre fe y razón se da en relación con el significado de Dios y del alcance de las proposiciones que de este concepto de dan; así pues, se discute dentro del área de la semántica si las proposiciones sobre Dios se refieren a verdades o solamente a actitudes subjetivas y a simbolismos; finalmente,

3. La oposición entre fe y razón se debate en los ámbitos de la epistemología, es decir en la posibilidad o no de poseer un conocimiento de Dios a través tanto de la razón como de la fe (Audi, 1991: pp. 213-214).

A estas discusiones (que van desde los debates patrísticos sobre la relación entre filosofía y teología hasta las ardientes disputas de los ilustrados sobre los argumentos clásicos de la existencia de Dios), se deben agregar los esfuerzos de muchos intelectuales por tratar de encapsular la noción de fe entre los límites de la misma razón y ya no solo en anteponer un concepto frente a otro como fuentes legítimas para acceder al conocimiento de la existencia de Dios, su significado y la posibilidad de abrir canales de inteligibilidad entre Dios y la humanidad.

Para abordar el conjunto de transformaciones del concepto de fe, desde Pablo, es necesario analizar tanto la forma en que Karl Rahner funda parte de su discurso teológico en un concepto renovado de fe a partir de sus contextos históricos, sociales, culturales y religiosos; así como la forma en que sobre este concepto fundamenta otras posibilidades discursivas, principalmente aquellas que se relacionan con la actualidad de los dogmas de fe cristiana que representa: las condiciones de posibilidad para el acceso a la realidad divina, la respuesta ética de la humanidad ante tales condiciones de posibilidad y las nuevas relaciones entre la teología, las ciencias y otras manifestaciones culturales, viendo estas relaciones desde la fe.

Karl Rahner es representante de una generación de pensadores posconciliares que responden a los cambios sociales, económicos y culturales de la Europa de posguerra; un grupo cuyo legado intelectual se enmarca sobre todo en la reconstitución del diálogo entre iglesia y modernidad en campos tan variados como el pensamiento filosófico de la religión y la espiritualidad, la teología y sus métodos, las iglesias organizadas, la diversidad, la ética y la fe propiamente dicha (Marmion y Hines, 2005, pp. 1-10). Según muchos, Rahner es quizá el más importante y, para nuestro criterio, uno a partir de cuyo trabajo podemos reconfigurar la forma en que el concepto de fe se ha ido ajustando a los procesos de continuidad y discontinuidad contemporáneos. Efectivamente, el concepto de fe subyace como primera fuente discursiva en muchos de los textos de Rahner, a partir siempre del debate entre fe y razón, debate que lo funda pero que, por otro lado, intenta superar dejándolo atrás en favor de la supremacía del concepto de fe, y revaluando los términos de la relación entre teología y otras ciencias humanas.

\section{La Fe como Experiencia}

El mejor inicio para comprender mejor la forma en que el trabajo de Karl Rahner se incrusta en una tradición específica dentro de la iglesia católica y el pensamiento teológico cristiano del siglo XX, al tiempo que pretende ir más allá de esta tradición para modificarla, actualizarla y transformarla en cierta medida, sería quizá analizar las tres principales fuentes de influencia para su proyecto teológico, los conceptos capitales que se desarrollan a partir de esa influencia y a qué discursos pretende enfrentarse.

Tres son los espacios desde donde Rahner se forma como teólogo y desde donde se nutre su sistema de pensamiento teológico, espacios sobre los cuales todos los comentaristas están indudablemente de acuerdo:

1. Su formación como jesuita pero, sobre todo, el influjo del pensamiento de su fundador Ignacio de Loyola, al que regresa cada vez que pretende desarrollar sus principales tesis y en el que encuentra inspiración para guiar la estructura de sus textos más representativos; 
2. La inclinación de su hermano Hugo, también jesuita, cuyos trabajos, sobre todo en relación con la vida y obra de Loyola y la relación entre iglesia y estado a lo largo de la historia del cristianismo, tuvieron un gran impacto en la visión de iglesia a la cual Rahner dedicaría mucha de su obra. Karl, además, adquirió de su hermano la disciplina académica que también caracteriza su trabajo;

3. El tercer espacio de influencia de Rahner está compuesto por tres fuentes adicionales:

a. por un lado, el pensamiento de Santo Tomás de Aquino, el teólogo más influyente de la escolástica incluso hasta principios del siglo XX;

b. por otro lado, el idealismo clásico alemán, sobre todo el trabajo de Immanuel Kant;

c. principalmente, el trabajo del teólogo francés jesuita Joseph Maréchal, uno de los principales intérpretes de la teología tomista a la luz del idealismo alemán, y uno de los principales exponentes de la nueva teología, un movimiento reformista y opuesto al neo-escolasticismo predominante a principios del siglo XX (Marmion y Hines, 2005).

De estos tres espacios de influencia -que marcan el trabajo de Rahner desde sus propios inicios- desarrolla la mayoría de sus principales conceptos, muchos de los cuales reinterpretan el concepto de fe que, para finales del siglo XIX y principios del XX, se encontraba limitado básicamente a dos esferas: por una parte, la fe en oposición con el concepto de razón (sobre todo en relación con el positivismo y el utilitarismo dominantes) y, por otra parte, la fe como el conjunto de dogmas sobre los cuales se fundamenta la correcta interpretación de las escrituras, los misterios, las formulaciones religiosas y los manuales y textos de formación religiosa. Él, al igual que los representantes de la nueva teología, pretende desligarse de estas dos esferas de interpretación del concepto de fe y regresa a las bases del pensamiento cristiano temprano para así readecuar los sistemas de fe hacia contextos más contemporáneos y, si se quiere, más plurales. Para Rahner, el concepto de fe, más bien, se encuentra en la base y no de los conflictos inherentes entre este concepto y las facultades de la razón, ni se limita a un conjunto de fórmulas magisteriales (que en definitiva Rahner tampoco desecha del todo, como veremos más adelante), sino que se relaciona con al menos tres elementos que serán relevantes a lo largo de su vida y obra:

1. Su concepto de fe se basa en la experiencia de la gracia de Dios, entendiéndola como el acto de Dios de auto-comunicarse y la fe como esa facultad inmanente al ser humano para poder experimentar la auto-comunicación del ser supremo; así, la fe en Rahner es parte de un sistema de carismas formado principalmente por la fe, el amor y la esperanza, trilogía que sirve de plataforma para el desarrollo de su sistema de práctica espiritual (Rahner, 1984);

2. La fe es una condición de posibilidad para la existencia humana; no es una decisión o posición ética o religiosa adoptada en oposición a la razón para poder dar explicaciones a fenómenos que se escapan a la explicación racional, sino que la propia existencia humana esta ineludiblemente conectada a la fe como su principal condición de posibilidad, y el medio por el cual el ser humano puede darle respuestas al total de su existencia;

3. La fe, como condición de posibilidad de la existencia humana y plataforma que permite la experiencia de la auto-comunicación de Dios, es histórica en tanto forma parte esencial de la posibilidad de la existencia y la experiencia humana por lo que, para Rahner, un sistema de creencias basado en la fe debe conducir al ser humano a las respuestas que necesita en su nivel de existencia espacio temporal propio si dicho sistema pretende tener actualidad (Rahner, 1984, pp. 37-43). 
A partir de estos tres conceptos (la auto-comunicación de Dios, la experiencia de Dios como condición de posibilidad de la existencia humana, y la historicidad del ser humano), Rahner problematiza y resemantiza la noción de fe hundiendo dicho concepto en las reinterpretaciones que hace de su herencia y tradición cristianas, sobre todo (tal y como lo hacen otros representantes de la nueva teología) en el estudio de fuentes tradicionales de pensamiento cristiano tales como las padres de la iglesia, Santo Tomás de Aquino, y el Nuevo Testamento, así como a partir de los espacios de influencia en los que se forma como teólogo, incluyendo sobre todo, el movimiento jesuita, el idealismo alemán y la nueva teología. A su vez, se enfrenta a una iglesia desgastada, la cual considera lejana de las nuevas realidades que afectan al ser humano y cada vez más distante de las necesidades y preguntas que aquejan a las sociedades que le son contemporáneas.

\section{El Proyecto Teológico de Rahner}

Debido a la gran producción teológica, social, filosófica y magisterial de Karl Rahner es sumamente difícil determinar la línea de base sobre la cual se puede montar todo su sistema teológico. Es incluso discutible el describir su obra como un sistema (Kilby, 2007). Aunque no es esa la idea en este momento, sí es posible determinar qué parte del proyecto teológico de Rahner puede ser reconstruido a partir de la resemantización que él hace del concepto de fe y cuyos elementos más importantes hemos abordado en la sección anterior. Nos toca ahora profundizar un poco más en la relevancia de dicha resemantización para comprender de mejor forma el papel que quiere darle al concepto de fe en su discurso teológico en general y poder detectar una forma esencialmente diferente de hablar sobre la fe. Ya hemos visto cómo esta reformulación tiene raíces en un movimiento de reforma dentro del catolicismo, principalmente, alemán y francés, conocido como nueva teología. Ahora veremos cómo Rahner fundamenta muchos de sus aportes innovando la forma en que el concepto de fe funciona a la base de la radicalización hecha por muchos otros pensadores, sobre todo en relación con una nueva práctica espiritual (Lehmann y Raffelt en Rahner, 1984, pp. ix-xv).

En síntesis, el modelo de fe de Rahner puede ser entendido a partir de los siguientes elementos:

1. La realidad divina es tanto del orden de lo ontológico como del orden de lo simbólico y epistemológico;

2. El ser humano le da sentido al mundo y a la realidad a partir de sus facultades trascendentes pero también a partir de sus condiciones históricas y biográficas;

3. La existencia divina y la existencia humana tienden una hacia la otra; la primera por su naturaleza de auto-comunicación gratuita; la segunda como respuesta de fe hacia el misterio que es en sí la existencia divina;

4. Las formas que tiene el ser humano para referirse al misterio de Dios son del orden de sus posibilidades históricas; esto es, a partir de las formas que tiene el ser humano para referirse a sí mismo. Desde esta perspectiva la teología es además una antropología y dice más del ser humano que la construye que de la existencia de Dios;

5. Cualquier intento de integrar el pensamiento religioso debe hacerse en respuesta a la historicidad del ser humano y a la actualidad del acto de auto-comunicación de Dios dentro de esas coordenadas de historicidad humana.

A partir de estas unidades, se reconstruyen tres de las grandes líneas de desarrollo teológico que forman parte de un todo del pensamiento de Rahner y que son de gran importancia para el concepto de fe resemantizado por él y para los propósitos de este trabajo; el primer subsistema es el misticismo rahneriano por medio del cual describe las bases para la experimentación de la realidad divina y las posibilidades de comunicación esenciales entre Dios y el ser humano. Para 
este subsistema, al cual Rahner dedica tres de sus obras más emblemáticas (Espíritu en el Mundo, El Oyente de la Palabra y Encuentros con el Silencio), elabora tres argumentos: el primero de ellos se basa en la premisa de que Dios (o la realidad divina) tiende a la auto-comunicación, ya que Dios es esencialmente conocimiento, o por mejor decir, es la base de todo conocimiento posible que, a diferencia de Kant, no depende solamente de intuiciones sensibles.

Therefore being and knowing are related to each other because originally, in their ground, they are the same reality. This does not imply anything less than that being as such, to the extent that it is being, is knowing; it is knowing in original unity with being, hence it is a knowing of the being who the knower is. Being and knowing constitute an original unity; that is: to the nature of being belongs a relation to knowing with regard to itself. (Rahner, 1994, pp. 29-30)

A este argumento llega en Espiritu en el Mundo donde Rahner intenta reconocer la posibilidad de una metafísica reinterpretando y estableciendo un diálogo entre Kant y Santo Tomás de Aquino en el que ambos autores se corrigen mutuamente, ya que Dios es esencialmente conocimiento, su ser (su metafísica) tiende a comunicarse, revelarse y esta es la base misma de la existencia humana en tanto ser sensible y racional (Rahner, 1968).

El segundo argumento se refiere al ser humano y su respuesta existencial a la revelación de la divinidad. En este sentido, Rahner define al ser humano como esencialmente espíritu por lo que está predestinado a ser oyente de la revelación. Según el autor, el ser humano y su intuición hacia lo cognoscible lo predetermina a concluir tanto lo específico de lo que conoce como la fuente infinita de ese conocimiento (Rahner, 1994). La acción humana de darle sentido al mundo a partir de la intuición en primera instancia y luego, a partir de su capacidad racional implica una tendencia hacia el misterio de lo total del conocimiento; tendencia que lo hace postular la idea de lo infinitamente cognoscible que en sus términos es Dios ser y conocimiento. Su revelación es solamente posible si existe esta tendencia humana hacia el conocimiento:

A divine revelation is possible only if we ourselves, the subjects to whom it is addressed, offer it an a priori horizon within which something like the revelation may occur. And only if this horizon is absolutely unlimited will no law or restriction be imposed from the start on a possible revelation concerning what might and should possibly be revealed. A revelation that is to unveil the depths of Divinity and that is basically the first moment of the invitation addressed to humanity to share in the life of almighty God, is conceivable and possible only if the human person is understood as spirit, i.e., as transcendence toward being pure and simple. (pp. 53-54).

Por consiguiente, existe la posibilidad fundamental de poder establecer un vínculo de comunicación entre lo divino y lo humano, ya que es la condición de posibilidad de la acción humana, producto de un sujeto que no es más que espíritu que conoce. Esta es la base para la espiritualidad práctica o misticismo rahneriano.

El segundo subsistema es la antropología rahneriana. Si anterior intenta rescatar la noción de la posibilidad de desarrollar una metafísica y su relación con la revelación de Dios, la antropología rahneriana le da el marco de posibilidad a esa metafísica dentro del conjunto de disciplinas o acciones humanas que intentan lidiar con el problema fundamental del ser humano: sí mismo. Es por eso que para Rahner, tanto la metafísica como la teología son en realidad diferentes antropologías. Es esta dimensión la piedra angular sobre la cual Rahner articula la conexión entre filosofía y teología entre el ser humano y Dios.

Con ello, ya en este primer paso se da un peculiar entrelazamiento entre filosofía y teología. Los presupuestos que aqui han de pensarse se refieren a la esencia del ser humano. Se refieren a su esencia constitutiva siempre históricamente, la cual, por tanto, se halla también sujeta a la confrontación con el cristianismo (como gracia y mensaje histórico) (Rahner, 1979, p. 43).

La teología de Rahner es histórica, ya que para él el ser humano siempre se encuentra 
atravesado por su realidad histórica, elemento que condiciona la forma en que accede al misterio de la revelación de Dios y, al mismo tiempo, al ser dirigida hacia el ser humano, también históricodependiente. La interpretación que el ser humano pueda hacer de dicha revelación (auto-comunicación de Dios), dependerá por consiguiente, de su historicidad, condición socio-cultural y del condicionamiento del pensar y actuar humano dentro de los límites del mundo de la vida humana. Es por eso además, que el contenido de la revelación debe también actualizarse según las condiciones de posibilidad humana para acceder a dichos contenidos revelados. Para Rahner la relación entre Dios y el ser humano no contempla, en primera instancia, un cuestionamiento moral sino existencial-ontológico (p. 42).

El tercer subsistema rahneriano referente a la fe es el ético-eclesiológico, el cual está íntimamente relacionado con los subsistemas anteriores. Por un lado, ya que la divinidad se revela o comunica gratuitamente al ser humano, Rahner define la fe esencialmente como la respuesta del ser humano a este "acercamiento" cognoscitivo de Dios hacia el ser humano. Esta respuesta se da en diferentes grados, siendo los primeros grados esencialmente no conscientes o voluntarios, sino parte de la condición de posibilidad para la existencia humana. Pero, los grados superiores si requieren de una acción racional y volitiva por parte del ser humano, esto es, una respuesta mayormente consciente, una aceptación de esa condición de comunicación entre ser humano y divinidad. Este paso de una fe anónima a una explicita (Donovan en Marmion y Hines, 2005, pp. 94-95) es la base de la acción ética del ser humano en relación con lo divino y en relación con su propia condición humana y depende y justifica la libertad humana:

En tanto el hacia dónde y el de dónde de la trascendencia no puede ser indiferente para el sujeto como cognoscente, en igual medida y por la misma razón la libertad tiene que habérselas con Dios originaria e inevitablemente. Libertad es libertad del sí o no a Dios y en ello y por ello libertad para sí misma. (Rahner, 1979, p. 129).
Así pues, el desarrollo de una respuesta positiva a Dios, de una ética humana es producto directo de la condición trascendente de la comunicación divina y humana.

Por otro lado, los condicionamientos socioculturales e históricos de la existencia humana obligan a que los seres humanos, en su relación trascendente y existencial con Dios, organicen el conocimiento resultante en comunidades religiosas responsables hecho que, por consiguiente, obliga a la iglesia a adaptarse a esas mismas condiciones socio-históricas.

\section{Fe e Iglesia: La iglesia y sus desafíos históricos}

Para Rahner, la iglesia, entendida como asociación de creyentes y organizada, está claramente diferenciada en términos históricos y su orientación futura debe basarse en el concepto de fe, en particular, (entendida la fe como respuesta humana histórica a la auto-comunicación de Dios en la historia del ser humano), así como en los demás carismas esenciales en general (estos son amor y esperanza). Por lo tanto, es menester de la iglesia entrar en periodos de revisión de sus elementos fundamentales. Rahner divide su análisis eclesiológico en tres momentos históricos:

1. El pasado, representado por la neo-escolástica y la apologética dominante durante, sobre todo, el siglo XIX, caracterizada por un excesivo dogmatismo y aferrada a conceptos inflexibles y tradicionales;

2. El presente, caracterizado por periodos de inmenso dolor y desencanto humano, sobre todo después de los acontecimientos asociados con las grandes guerras europeas, la guerra fría y las condiciones de inestabilidad política global durante la segunda mitad del siglo XX; este presente es traducido por Rahner como una época de grandes desafíos, sobre todo en lo referente a la forma en que la iglesia cristiana debe comunicarse con sociedades cada vez más diversas y escépticas, y a la existencia de diferentes modos de 
organización social que no necesariamente tienen relación con la iglesia;

3. Un futuro de la iglesia sobre el cual Rahner diserta abundantemente desde su cátedra universitaria en la formación de futuros teólogos así como al repasar los elementos constitutivos de la dogmática cristiana. (cita bibliográfica)

Curiosamente, la iglesia del futuro tal y como la describe Rahner depende de un retorno a los inicios simples y esenciales de las primeras comunidades cristianas, pequeñas comunidades unidas por la fe de sus miembros, y de estas pequeñas comunidades de creyentes deben erigirse de nuevo las grandes estructuras eclesiológicas de la fe.

The Church of the future will be one built from below by basic communities as a result of free initiative and association. We should make every effort not to hold up, but to promote this development and direct it on to the right lines. To understand what this means, we must recall what was said in the first part of these reflections: concretely and socially, the Church will no longer exist as formerly through the mere persistence of her office, of her socially firm structures, and through an awareness of being taken for granted by public opinion, recruiting new members simply because the children adopt and maintain the life-style of their parents and are baptized and indoctrinated by the Church. The Church will exist only by being constantly renewed by a free decision of faith and the formation of congregations on the part of individuals in the midst of a secular society bearing no imprint of Christianity. (Rahner, 1972, p. 108)

De manera que, para Rahner, el futuro de la iglesia no radica en la perpetuación de la tradición y la salvaguarda de los dogmas, ni siquiera en una fuerte constitución del dominio papal (romano) (Rahner, 1972, pp. 53-60), sino en la fe como respuesta libre de seres humanos dispuestos a aceptar la invitación de comunicación divina, siempre a partir de la realidad concreta de dichos seres humanos. Una vez que este movimiento consciente y libre de aceptación de la revelación divina, las comunidades de fe y sus líderes deben ser recibidos y reconocidos por la estructura eclesial principal la cual en su totalidad se adapta a todo el pluralismo esencial asociado a estas comunidades de fe (p. 109).

Esta inversión, según la cual Rahner propone que la gran iglesia sea afectada por las pequeñas comunidades de fe para así buscar su adaptación, en lugar de que la gran iglesia sea la que dicte las líneas de pensamiento de todos sus miembros, es lo que llama "democratización de la iglesia" o "iglesia democratizada" (p. 119). En esta iglesia democratizada, Rahner concibe una mayor participación de los laicos en diferentes procesos de toma de decisiones de la iglesia. Igualmente, este desarrollo de la base a la cima (de las iglesias de base al papado) funda las bases para la construcción de una iglesia más socio-crítica es decir, capaz de llevar a cabo con éxito su responsabilidad social en el mundo más allá de ser una institución de caridad o servicio social.

\section{Fe en Cristo en Rahner}

Igual que en Pablo, el sistema de pensamiento de Rahner basado en el concepto de fe es esencialmente cristológico, pero no es cristocéntrico, ya que su núcleo es doble; por un lado, depende fuertemente de la realidad de la acción de Dios en el mundo y por otro, de la situación de ese accionar en coordenadas históricas; esto quiere decir que, ante todo, el sistema de Rahner es teo-antropocéntrico.

La cristología rahneriana se basa, esencialmente, en la resemantización del concepto fe en relación con la tendencia trascendente de la comunicación Dios-seres humanos en lo concreto de las coordenadas histórico-culturales y sociales del ser humano. En relación con este diagrama de revelación y la correspondiente respuesta humana a esta acción divina dentro de su misma historicidad (historia de la revelación), Rahner plantea los siguientes argumentos esenciales:

1. La auto-comunicación divina es histórica y por ende, dicha historia puede ser reconstruida. 
2. Dicha historia de la revelación alcanza su punto culminante esencialmente en el acto de auto-comunicación divina en la realidad histórica del ser humano en la encarnación de Dios en el mundo.

3. La encarnación de Dios en el mundo es la máxima expresión de comunicación divina y el punto de mayor acercamiento entre la tendencia de auto-comunicación de Dios y la capacidad humana de interpretar desde su realidad histórica dicha comunicación. Nunca antes y ni después, se puede llegar a un mayor nivel de comunicación.

4. Esta comunicación máxima tiene tres elementos: la encarnación de lo enunciado y comunicado (Dios); la realidad humana de Cristo (Dios vivo y en estado definitivo); la realidad de Jesús, como el ser humano que contempla a Dios. La relación Dios, Cristo y Jesús son entonces una unidad en la encarnación de Dios, como máximo grado de comunicación Dios-humanidad.

5. El hecho llamado Cristo es la única evidencia realmente aprehensible para el ser humano de la historia general de la revelación.

Jesucristo es para Rahner el punto de mayor acercamiento entre el acto gratuito de comunicación de Dios con la humanidad y la capacidad humana de comprender este acto de comunicación. Este acto entre Dios y el mundo por medio de la realidad histórica de Jesús, es considerado por Rahner como una concepción evolutiva del mundo (Rahner, 1979, p. 216), y justifica el hecho de que es Jesucristo el equivalente al salvador absoluto.

Sin embargo, no es Jesucristo el elemento central de la teología de la revelación de Rahner, sino solo el hecho histórico a través del cual dicha revelación puede ser mejor aprehendida por el ser humano. En este sentido, Jesús representa la humanidad "agraciada" por el hecho trascendental de la comunicación divina, y solo en tanto es depositario de la encarnación de Dios. Por esta razón, Rahner considera la posibilidad de que haya "cristianos anónimos", esto es, seres humanos que, a pesar de que no son conscientes de la realidad de la encarnación divina en Jesús, aun así participan de la tendencia trascendental de la auto-comunicación y divina y la respuesta, también trascendental, del ser humano como espíritu. Este aspecto de la teología de Rahner es polémica en dos sentidos: En primer lugar, a pesar de que desarrolla una cristología bien cimentada, su teología no es cristocéntrica y su concepto de fe no depende directamente del suceso particular de Jesús Cristo, sino básicamente del hecho de la posibilidad de comunicación Dios-humanidad, se sea consciente de esto o no; y segundo, la pretensión de Rahner de incluir a todos los seres humanos y su práctica espiritual desde una óptica netamente cristiana; de ahí la polémica alrededor de su concepto del cristiano anónimo.

\section{Resumen}

El trabajo de Rahner, altamente extenso y complejo, incluso difícil de concebir como un todo sistemático, se caracteriza por conceptos claves en cuya base se encuentra una resemantización del concepto de fe, el cual hasta ese momento se encontraba encajonado dentro de los límites de un debate milenario en contra del influjo de la razón. Este concepto de fe contiene las siguientes características cardinales:

1. Describe una realidad esencialmente experiencial relativa a las condiciones mismas de la existencia humana y una forma de describir un cierto tipo de experiencia humana.

2. El tipo de experiencia basamental que se monta sobre el concepto de fe es aquella a través de la cual se es permitido conocer en tanto ser espiritual y cognoscente.

3. Lo que se experimenta y conoce por medio de la fe es la tendencia de auto-comunicación de lo divino en tanto es ser y conocimiento a la vez. 
4. Es esencial y primeramente (en los términos más simples) la opción consciente de un ser humano por escuchar lo que la divinidad quiere comunicarle en su impulso de autocomunicación primordial.

5. La fe describe, por ende, la experiencia mística por excelencia en la que me es posible comunicarme con Dios.

6. Es fundamentalmente teocéntrico, ya que el acto de comunicación al cual accedo por fe es el acto comunicativo de Dios; sin embargo, en la historia de dicha comunicación (revelación), llegó a su punto de mayor posibilidad en la encarnación de Dios en Jesucristo. Por lo tanto, su punto de mayor afirmación y aprehensión es cristológica.

7. Está determinado por la historicidad que forma parte intrínseca de la realidad humana. La fe es parte de un sistema tanto trascendental (en la medida que es condición necesaria) como existencial (en tanto se materializa en coordenadas de tiempo y espacio determinados).

8. No se antepone a la razón sino que es punto de partida para un diálogo abierto e informativo entre diferentes disciplinas del saber humano.

9. Forma parte de un conjunto carismático formado por la fe, el amor y la esperanza, y debe ser analizado siempre en relación con los otros elementos de este conjunto.

\section{Referencias}

Allen, J. Debating Karl Rahner and Hans urs Von Balthasar; Interview with David Schindler; Appointments in the Roman Curia; Archbishop Laurent Monsengwo of Kisangani. En www.ratzingerfanclub.com/Balthasar

Allik, T. (1982). God and the Unconscious: On Karl Rahner, Justification by Faith, and the Freudian Unconscious. Yale University. Estados Unidos.
Audi, R. (1991). Faith, Belief and Rationality. Philosophical Perspectives, Vol. 5, Philosophy of Religion. pp.213-239.

Bramwell, B. The Faith-Culture Dialogue: Karl Rahner and Hans Urs von Von Balthasar. Franciscan University. http://www.cssronline.org/CSSR/Archival/2002/Article-Bramwell.pdf

Costello, E. Father Karl Rahner, S.J. Brilliant Theologian, Prodigious Writer. En www.costello.au.com/articles/11. html

Dunn, James D.G. (1998). The Theology of Paul the Apostle. T\&T Clark. Edinburgh.

Eichholz, Georg. (1977). El Evangelio de Pablo: Esbozo de la teología paulina. Ediciones Sígueme, Salamanca.

Faith and Paul's Theology of the Law/Gospel Contrast in Galatians 3:9-12. En www.geocities.com/athens/delphi/8449/gospelandlaw.html

Gidley, J. James and Justification by Faith. En http://www. opc.org/new_horizons/NH05/02a.html

Ginascol, F. (1959). The Question of Universals and the Problem of Faith and Reason. The Philosophical Quarterly, Vol. 9, No. 37. Oct., pp. 319-329.

Helm, P. (1973). Locke on Faith and Knowledge. The Philosophical Quarterly, Vol. 23, No. 90. Jan. pp. 52-66.

James, W. (1927). Reason and Faith. The Journal of Philosophy, Vol. 24, No. 8. Apr. 14, pp. 197-201.

Jordan, P. (1972). La Dimensión Divina: Reflexiones sobre la Fe. Herdes, Barcelona.

Kant, I. (2007). La Religión Dentro de los Límites de la Mera Razón. Alianza Editorial, España.

Kilby, K. (2007). Rahner. The Blackwell Companion to Modern Theology. Oxford.

Kilby, K. (1997). Karl Rahner. A brief introduction. Herder and Herder. New York.

Marmion, D y Hines, M. Eds. (2005). The Cambridge Companion to Karl Rahner. Cambridge University Press. England.

McGrath, A. Ed. (2007). The Christian Theology Reader. Blackwell Publishing. England.

Olsen, C. (2007). Act and Event in Rahner and Von Balthasar: A Case Study in Catholic Systematics. The Dominican Council. Blackwell Publishing Ltd., Oxford.

Rahner, K. (s.f.) Cambios en la Iglesia. Ediciones Paulinas, Madrid. 
(1994). Hearer of the Word. Continuum. New

York.

(1984). The Practice of Faith: A Handbook of Contemporary Spirituality.

Crossroad. New York.

(1979). Curso fundamental Sobre la Fe. Herder, Barcelona.

. (1974). Cambio Estructural de la Iglesia. Eds. Cristiandad, Madrid.

(1972). The Shape of the Church to Come. SPCK. London.

(1970). J. Razinger. Revelación y Tradición. Herdes, Barcelona.

(1968). Spirit in the World. Herder and Herder. New York.
(1967). Filosofía y Teología. Escritos sobre Teología, Tomo VI, p.90. Taurus Ediciones, Madrid.

(1967). Ecrits Théologiques. Tome VIII. Textes et Études Théologiques. Paris.

et al. (1970). La Reforma que Llega de Roma. Rotativa, España.

Scanlon, R. Non-Infallibility: The Papacy And Rahner. En www.catholicculture.org/library/view.cfm?recnum $=4210$

Schilpp, P. (1924). A Rational Basis Demanded for Faith. The Journal of Philosophy, Vol. 21, No. 8, pp. 209-212.

Theissen, Gerd (2002). La Redacción de los Evangelios y la Política Eclesial: En enfoque socio-retórico. Editorial Verbo Divino. España

Wong, N. Karl Rahner's Concept of the 'Anonymous Christian':An Inclusivist View of Religions. En www.ttc. edu.sg/csca/CS/2001-Apr/Norman\%20Wong.pdf 